\title{
Cornering variants of the Georgi-Machacek model using Higgs precision data
}

\author{
Dipankar Das ${ }^{1,2, *}$ and Ipsita Saha ${ }^{3, \dagger}$ \\ ${ }^{1}$ Department of Physics, University of Calcutta, 92 Acharya Prafulla Chandra Road, Kolkata 700009, India \\ ${ }^{2}$ Department of Astronomy and Theoretical Physics, Lund University, 22100 Lund, Sweden \\ ${ }^{3}$ Istituto Nazionale di Fisica Nucleare, Sezione di Roma, Piazzale Aldo Moro, 2, 00185, Roma, Italy
}

(Received 7 July 2018; published 12 November 2018)

\begin{abstract}
We show that in the absence of trilinear terms in the scalar potential of the Georgi-Machacek model, heavy charged scalars do not necessarily decouple from the $h \rightarrow \gamma \gamma$ decay amplitude. In such scenarios, measurement of the Higgs to diphoton signal strength can place stringent constraints in the parameter space. Using the projected precisions at the High Luminosity LHC (HL-LHC) and the ILC, we find that the upper bound on the triplet vacuum expectation value can be as low as $10 \mathrm{GeV}$. We also found that when combined with the theoretical constraints from perturbative unitarity and stability, such variants may be ruled out altogether.
\end{abstract}

DOI: $10.1103 /$ PhysRevD.98.095010

The discovery of a Higgs-like scalar at the Large Hadron Collider (LHC) [1,2], has initiated a deeper investigation into its origin. It is now time to settle whether this new particle is the Higgs boson as predicted by the standard model (SM), or it is a Higgs-like particle stemming from a more elaborate construction beyond the SM (BSM). In view of the latter intriguing possibility, recent years have seen a growing interest in BSM scenarios with an extended Higgs sector, where the scalar boson observed at the LHC is only the first to appear in a series of many others to follow. The Georgi-Machacek (GM) model [3-5] constitutes one of the simplest examples of this category and therefore has received a lot of attention in recent times.

The SM extended by a scalar triplet only, which goes by the name of Higgs triplet model (HTM) does not preserve the custodial symmetry at the tree-level. Consequently, the vacuum expectation value (VEV) of the triplet is restricted to be below $(\mathcal{O})(10 \mathrm{GeV})$ [6] so that the value of the electroweak $\rho$-parameter remains consistent with the experimental fits. In the GM model, two scalar triplets (one real and one complex) are added in such a way that the custodial symmetry is preserved at the tree-level in the limit when the two triplets assume a common VEV, $v_{t}$, after the spontaneous symmetry breaking (SSB). Therefore, unlike in the HTM, the constraint on $v_{t}$ arising from the electroweak $\rho$-parameter is lifted in the case of the GM model.

\footnotetext{
dipankar.das@thep.lu.se

ipsita.saha@roma1.infn.it
}

Published by the American Physical Society under the terms of the Creative Commons Attribution 4.0 International license. Further distribution of this work must maintain attribution to the author(s) and the published article's title, journal citation, and DOI. Funded by SCOAP.
However, since the charged scalars couple to the SM fermions with strengths proportional to $v_{t}$, upper bound on $v_{t}$ can still be placed using the flavor data when the charged scalars are sufficiently light. But such an upper bound gets diluted very quickly with increasing charged scalar masses to the extent that for charged scalar masses of $(\mathcal{O})(1 \mathrm{TeV})$ one can allow $v_{t}$ to be as large as $60 \mathrm{GeV}[7,8]$. Our motivation in this paper is to put constraints on the triplet VEV, $v_{t}$, even when the nonstandard scalars are super heavy. We will employ the Higgs data to achieve this purpose. As a first step, we shall reanalyze the scalar potential of the GM model and identify the lightest $C P$-even scalar $(h)$ in the spectrum as the $125 \mathrm{GeV}$ resonance observed at the LHC. Then we compute the $V V h(V=W, Z)$ and $\bar{f} f h(f$ denotes a generic fermion) in terms of the model parameters and compare them with the corresponding SM expectations taking into account current and future experimental accuracies. Admittedly, such studies have been performed earlier in the literature [9-20] but our current analysis differs in a crucial way in the sense that we highlight how the inclusion of $h \rightarrow \gamma \gamma$ signal strength measurement can significantly tighten the constraints in the parameter space. One key observation of our paper, which runs contrary to the common perception [12], is that the charged scalar contributions to the $h \rightarrow \gamma \gamma$ decay amplitude do not necessarily decouple in certain variants of the GM models. Consequently, for such variants, the bounds on the model parameters, especially $v_{t}$, extracted using the data for Higgs signal strengths will not get washed away even when the nonstandard masses are in the multi- $\mathrm{TeV}$ range. To our knowledge, this subtle but interesting feature has not been emphasized earlier in the context of GM models. 
We start by revisiting the scalar potential of the GM model. As stated earlier, GM model [3-5] extends the scalar sector of the SM by adding one real $S U(2)_{L}$ triplet $\xi$ and one complex $S U(2)_{L}$ triplet $\chi$ with hypercharges $Y=0$ and $Y=2$ respectively. The scalar fields of such a model can be concisely represented in the forms of a bidoublet $\Phi$ and a bitriplet $X$ as

$\Phi=\left(\begin{array}{cc}\phi^{0 *} & \phi^{+} \\ -\phi^{-} & \phi^{0}\end{array}\right), \quad X=\left(\begin{array}{ccc}\chi^{0 *} & \xi^{+} & \chi^{++} \\ -\chi^{-} & \xi^{0} & \chi^{+} \\ \chi^{--} & -\xi^{-} & \chi^{0}\end{array}\right)$.

In order to preserve the custodial symmetry, both the real and complex triplet should acquire the same VEV.
Accordingly, the VEVs of the scalar multiplets are defined by $\langle\Phi\rangle=\left(v_{d} / \sqrt{2}\right) \mathbb{1}_{2 \times 2}$ and $\langle X\rangle=v_{t} \mathbb{1}_{3 \times 3}$. Comparing the $W$ and $Z$ boson masses with their corresponding SM expressions, we obtain the formula for the electroweak (EW) VEV as

$$
v=\sqrt{v_{d}^{2}+8 v_{t}^{2}}=246 \mathrm{GeV} .
$$

Following the convention of Refs. [12,21-23], we now write down the most general scalar potential for the GM model as follows:

$$
\begin{aligned}
V(\Phi, X)= & \frac{\mu_{2}^{2}}{2} \operatorname{Tr}\left(\Phi^{\dagger} \Phi\right)+\frac{\mu_{3}^{2}}{2} \operatorname{Tr}\left(X^{\dagger} X\right)+\lambda_{1}\left[\operatorname{Tr}\left(\Phi^{\dagger} \Phi\right)\right]^{2}+\lambda_{2} \operatorname{Tr}\left(\Phi^{\dagger} \Phi\right) \operatorname{Tr}\left(X^{\dagger} X\right) \\
& +\lambda_{3} \operatorname{Tr}\left(X^{\dagger} X X^{\dagger} X\right)+\lambda_{4}\left[\operatorname{Tr}\left(X^{\dagger} X\right)\right]^{2}-\lambda_{5} \operatorname{Tr}\left(\Phi^{\dagger} \tau_{a} \Phi \tau_{b}\right) \operatorname{Tr}\left(X^{\dagger} t_{a} X t_{b}\right) \\
& -M_{1} \operatorname{Tr}\left(\Phi^{\dagger} \tau_{a} \Phi \tau_{b}\right)\left(U X U^{\dagger}\right)_{a b}-M_{2} \operatorname{Tr}\left(X^{\dagger} t_{a} X t_{b}\right)\left(U X U^{\dagger}\right)_{a b},
\end{aligned}
$$

where, $\tau_{a} \equiv \sigma_{a} / 2,(a=1,2,3)$ with $\sigma_{a}$ s being the Pauli matrices and $t_{a} \mathrm{~s}$ correspond to the generators of triplet representation of $S U(2)$ and are expressed as

$$
t_{1}=\frac{1}{\sqrt{2}}\left(\begin{array}{ccc}
0 & 1 & 0 \\
1 & 0 & 1 \\
0 & 1 & 0
\end{array}\right), \quad t_{2}=\frac{1}{\sqrt{2}}\left(\begin{array}{ccc}
0 & -i & 0 \\
i & 0 & -i \\
0 & i & 0
\end{array}\right), \quad t_{3}=\left(\begin{array}{ccc}
1 & 0 & 0 \\
0 & 0 & 0 \\
0 & 0 & -1
\end{array}\right) .
$$

The matrix $U$ appearing in the cubic terms of Eq. (3) is given by,

$$
U=\frac{1}{\sqrt{2}}\left(\begin{array}{ccc}
-1 & 0 & 1 \\
-i & 0 & -i \\
0 & \sqrt{2} & 0
\end{array}\right)
$$

In the limit when both the triplets receive the same VEV, we will have two independent minimization conditions, using which we can trade the bilinear coefficients, $\mu_{2}^{2}$ and $\mu_{3}^{2}$ in favor of $v_{d}$ and $v_{t}$ as follows:

$$
\begin{aligned}
& \mu_{2}^{2}=\frac{3}{2} M_{1} v_{t}-4 \lambda_{1} v_{d}^{2}-3\left(2 \lambda_{2}-\lambda_{5}\right) v_{t}^{2} \\
& \mu_{3}^{2}=\frac{M_{1} v_{d}^{2}}{4 v_{t}}+6 M_{2} v_{t}-\left(2 \lambda_{2}-\lambda_{5}\right) v_{d}^{2}-4\left(\lambda_{3}+3 \lambda_{4}\right) v_{t}^{2} .
\end{aligned}
$$

After SSB, the neutral component of the fields are expanded around the minima as

$\phi^{0}=\frac{v_{d}}{\sqrt{2}}+\frac{h_{d}+i z_{d}}{\sqrt{2}}, \quad \xi^{0}=v_{t}+h_{\xi}, \quad \chi^{0}=v_{t}+\frac{h_{\chi}+i z_{\chi}}{\sqrt{2}}$.
The mass terms in the scalar potential, then, can be diagonalized to obtain a custodial quintuplet $\left(\mathrm{H}_{5}^{++}, \mathrm{H}_{5}^{+}\right.$, $H_{5}^{0}, H_{5}^{-}, H_{5}^{--}$) with common mass $m_{5}$, a custodial triplet $\left(H_{3}^{+}, H_{0}, H_{3}^{-}\right)$with common mass $m_{3}$ and two custodial singlets $h$ and $H$ with masses $m_{h}$ and $m_{H}$ respectively. ${ }^{1}$ In particular, to diagonalize the $C P$-even scalar sector, we need to introduce an angle $\alpha$ which is defined as follows

$$
\left(\begin{array}{c}
H_{5}^{0} \\
H \\
h
\end{array}\right)=\left(\begin{array}{ccc}
1 & 0 & 0 \\
0 & \cos \alpha & -\sin \alpha \\
0 & \sin \alpha & \cos \alpha
\end{array}\right)\left(\begin{array}{ccc}
\sqrt{\frac{2}{3}} & -\sqrt{\frac{1}{3}} & 0 \\
\sqrt{\frac{1}{3}} & \sqrt{\frac{2}{3}} & 0 \\
0 & 0 & 1
\end{array}\right)\left(\begin{array}{c}
h_{\xi} \\
h_{\chi} \\
h_{d}
\end{array}\right) .
$$

In what follows, we assume $h$ to be the lightest among the $C P$-even scalars, which has been discovered at the LHC with mass $m_{h} \approx 125 \mathrm{GeV}$.

At this point it is instructive to count the number of independent parameters in the potential of Eq. (3). There are a total of nine parameters consisting of two bilinears $\left(\mu_{2}^{2}\right.$ and $\left.\mu_{3}^{2}\right)$, five quartic couplings $\left(\lambda_{i}, i=1, \ldots, 5\right)$, and two cubic couplings $\left(M_{1}\right.$ and $\left.M_{2}\right)$. Among these, $\mu_{2}^{2}$ and $\mu_{3}^{2}$ have

\footnotetext{
${ }^{1}$ For details of the diagonalization procedure we refer the reader to Refs. [12,22].
} 
already been traded in favor of $v_{d}$ and $v_{t}$ as in Eq. (6). The five quartic couplings can now be exchanged in favor of four physical masses, $m_{5}, m_{3}, m_{H}$, and $m_{h}$ and the mixing angle, $\alpha$. The relevant formulas connecting the $\lambda_{i}$-s with the physical masses and mixings are given below:

$$
\begin{aligned}
\lambda_{1}= & \frac{1}{8 v^{2} \cos ^{2} \beta}\left(m_{h}^{2} \cos ^{2} \alpha+m_{H}^{2} \sin ^{2} \alpha\right), \\
\lambda_{2}= & \frac{1}{12 v^{2} \cos \beta \sin \beta}\left(\sqrt{6}\left(m_{h}^{2}-m_{H}^{2}\right) \sin 2 \alpha\right. \\
& \left.-3 \sqrt{2} v \cos \beta M_{1}+12 m_{3}^{2} \sin \beta \cos \beta\right),
\end{aligned}
$$

$$
\lambda_{3}=\frac{1}{v^{2} \sin ^{2} \beta}\left(\sqrt{2} v \cos \beta \cot \beta M_{1}-3 \sqrt{2} v \sin \beta M_{2}\right.
$$$$
\left.-3 m_{3}^{2} \cos ^{2} \beta+m_{5}^{2}\right)
$$

$$
\begin{aligned}
\lambda_{4}= & \frac{1}{6 v^{2} \sin ^{2} \beta}\left(2 m_{H}^{2} \cos ^{2} \alpha+2 m_{h}^{2} \sin ^{2} \alpha-2 m_{5}^{2}\right. \\
& \left.+6 \cos ^{2} \beta m_{3}^{2}-3 \sqrt{2} v \cos \beta \cot \beta M_{1}+9 \sqrt{2} \sin \beta v M_{2}\right),
\end{aligned}
$$

$$
\lambda_{5}=-\frac{\sqrt{2} M_{1}}{v \sin \beta}+\frac{2 m_{3}^{2}}{v^{2}} .
$$

where, we have defined

$$
\tan \beta=\frac{2 \sqrt{2} v_{t}}{v_{d}} .
$$

Note that Eq. (9) will be extremely useful in translating the scalar couplings as well as our final results in terms of the physical parameters which are directly accessible at the experiments.

Before moving on to the main part of the paper, let us first examine the theoretical bounds coming from perturbative unitarity and stability of the scalar potential. The relevant constraints have been summarized in Refs. [12,21]. In Fig. 1 we display our main results in the limit $M_{1}$, $M_{2}=0$. It is quite intriguing to note that there arises a lower bound on $v_{t}(\gtrsim 27 \mathrm{GeV})$ from unitarity and stability. It is not very difficult to find a qualitative justification for such a lower bound as we will explain below.

Among the many unitarity constraints, we draw the reader's attention to the following ones $[12,21]$ in particular:

$$
\begin{gathered}
\left|16 \lambda_{3}+8 \lambda_{4}\right| \leq 8 \pi, \\
\left|4 \lambda_{3}+8 \lambda_{4}\right| \leq 8 \pi .
\end{gathered}
$$

After using Eq. (9) to substitute for the $\lambda_{i}$-s and putting $M_{1}$, $M_{2}=0$, the above conditions read

$$
\left|\frac{5}{3} m_{5}^{2}-5 m_{3}^{2} \cos ^{2} \beta+\frac{1}{3} m_{0}^{2}\right| \leq \pi v^{2} \sin ^{2} \beta,
$$

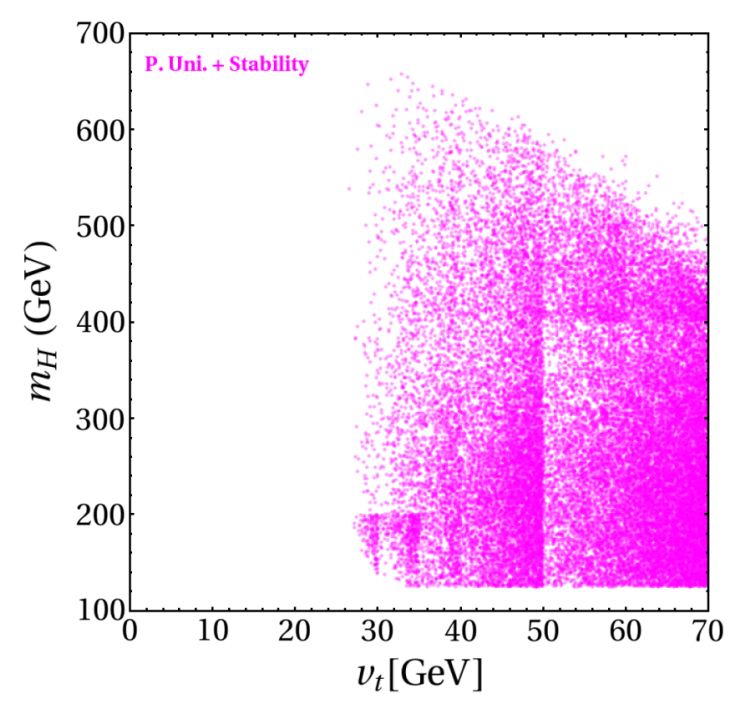

FIG. 1. Allowed region in the $v_{t}-m_{H}$ plane from perturbative unitarity and stability in the limit $M_{1}, M_{2}=0$. Note that a lower limit on $v_{t}$ emerges from these theoretical constraints.

$$
\left|\frac{1}{3} m_{5}^{2}-m_{3}^{2} \cos ^{2} \beta+\frac{2}{3} m_{0}^{2}\right| \leq 2 \pi v^{2} \sin ^{2} \beta
$$

where we have used the following abbreviation:

$$
m_{0}^{2}=m_{h}^{2} \sin ^{2} \alpha+m_{H}^{2} \cos ^{2} \alpha .
$$

Clearly, for very small $v_{t}$, i.e., $\sin \beta \rightarrow 0$, the following correlations will be imposed:

$$
\begin{gathered}
\frac{5}{3} m_{5}^{2}-5 m_{3}^{2}+\frac{1}{3} m_{0}^{2} \approx 0, \\
\frac{1}{3} m_{5}^{2}-m_{3}^{2}+\frac{2}{3} m_{0}^{2} \approx 0 .
\end{gathered}
$$

Moreover, the following unitarity conditions will also be relevant $[12,21]$ :

$$
\begin{aligned}
\left|x_{2}^{ \pm}\right| \equiv & \mid 4 \lambda_{1}-2 \lambda_{3}+4 \lambda_{4} \\
& \pm \sqrt{\left(4 \lambda_{1}+2 \lambda_{3}-4 \lambda_{4}\right)^{2}+4 \lambda_{5}^{2}} \mid \leq 8 \pi .
\end{aligned}
$$

Using the triangle inequality we may write,

$$
\begin{gathered}
\left|x_{2}^{+}+x_{2}^{-}\right| \leq 16 \pi \\
\Rightarrow\left|4 \lambda_{1}-2 \lambda_{3}+4 \lambda_{4}\right| \leq 8 \pi .
\end{gathered}
$$

In the above inequality, the terms involving $\lambda_{3}$ and $\lambda_{4}$ will give rise to the dominant contributions on the left-hand side for small $v_{t}$. Again, using Eq. (9) one can check that the limit $\sin \beta \rightarrow 0$ will entail the following correlation: 


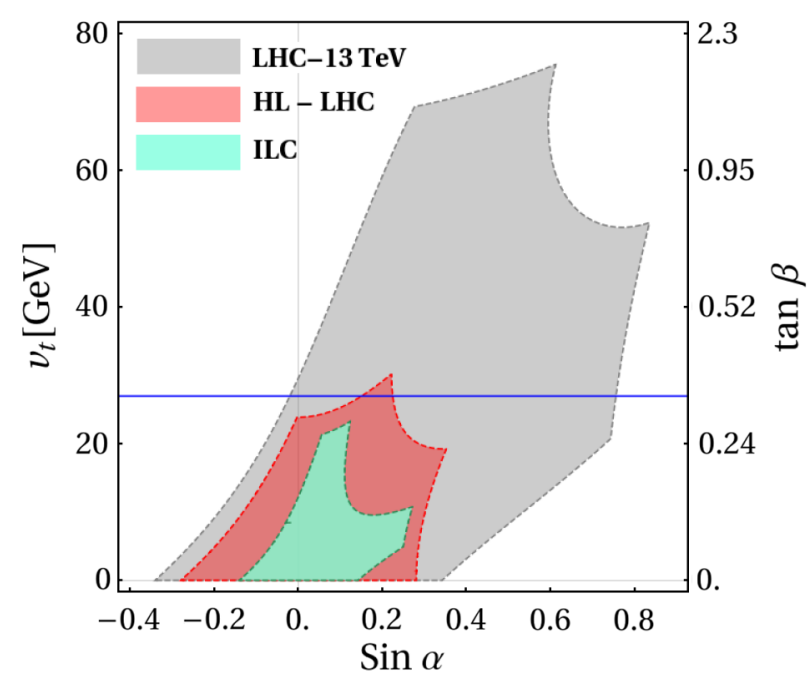

FIG. 2. Combined allowed regions at 95\% C.L. in the $\sin \alpha-v_{t}$ plane using the current [24] (shaded in gray) and projected [25] (shaded in red and green) accuracies in the measurements of $\kappa_{V}$ and $\kappa_{f}$. While extracting bounds using future precisions, the central values of all the $\kappa_{x}$ parameters have been assumed to be 1 , i.e., consistent with the SM. The horizontal line corresponds to the lower limit on $v_{t}$ arising from the theoretical constraints.

$$
\frac{5}{3} m_{5}^{2}-5 m_{3}^{2}-\frac{2}{3} m_{0}^{2} \approx 0
$$

It is easy to verify that no nontrivial solution set $\left\{m_{5}^{2}, m_{3}^{2}, m_{0}^{2}\right\}$ exists such that Eqs. (16), (17), and (20) are satisfied simultaneously. This explains why there should be a lower limit on $v_{t}$ (or equivalently, on $\tan \beta$ ) so that the correlations of Eqs. (16), (17), and (20) do not become too strong.

Let us now proceed by defining the Higgs coupling modifiers as:

$$
\kappa_{x}=\frac{g_{h x x}}{\left(g_{h x x}\right)_{\mathrm{SM}}}
$$

where $x$ stands for the gauge bosons and the massive fermions. In the GM models, these coupling modifiers can be calculated to be

$\kappa_{f}=\frac{\cos \alpha}{\cos \beta}, \quad(f=$ generic massive fermion $)$,

$\kappa_{V}=\cos \alpha \cos \beta+\frac{2 \sqrt{2}}{\sqrt{3}} \sin \alpha \sin \beta, \quad(V=W, Z)$.

Using the current limits on $\kappa_{f}$ and $\kappa_{V}$ [24], we display the $2 \sigma$ allowed region (light gray shade) in the $\sin \alpha-v_{t}$ plane in Fig. 2. In the same plot we also show how this allowed region will shrink if the Higgs data continue to drift towards the SM expectations with the projected accuracy at the HL-LHC $(2 \%)$ (red shade) and at the ILC $(<1 \%)$ (green shade) [25]. Clearly, with the projected accuracies at the HL-LHC and the ILC, we can hope to constrain $v_{t}$ to be smaller than $20 \mathrm{GeV}$.

A crucial observation of our paper is that the allowed region in Fig. 2 from $\kappa_{f}$ and $\kappa_{V}$ can be considerably reduced further by superimposing the constraints from $h \rightarrow \gamma \gamma$ even in the limit when the charged scalars are super heavy. To illustrate, let us first write down the effective $h \gamma \gamma$ coupling as follows:

$$
\mathcal{L}_{h \gamma \gamma}=g_{h \gamma \gamma} F_{\mu \nu} F^{\mu \nu} h
$$

where $F_{\mu \nu}=\partial_{\mu} A_{\nu}-\partial_{\nu} A_{\mu}$ is the usual electromagnetic field tensor. Now we define the $h \gamma \gamma$ coupling modifier as

$$
\kappa_{\gamma}=\frac{g_{h \gamma \gamma}}{\left(g_{h \gamma \gamma}\right)_{\mathrm{SM}}}
$$

whose analytic expression for the GM model can be written as ${ }^{2}$

$$
\kappa_{\gamma}=\frac{\kappa_{V} \mathcal{A}_{W}\left(\tau_{W}\right)+\sum_{f} Q_{f}^{2} N_{c}^{f} \kappa_{f} \mathcal{A}_{f}\left(\tau_{f}\right)+\kappa_{3} \mathcal{A}_{S}\left(\tau_{3}\right)+5 \kappa_{5} \mathcal{A}_{S}\left(\tau_{5}\right)}{\mathcal{A}_{W}\left(\tau_{W}\right)+\sum_{f} Q_{f}^{2} N_{c}^{f} \mathcal{A}_{f}\left(\tau_{f}\right)},
$$

where, $Q_{f}$ and $N_{c}^{f}$ denote the electric charge and the color factor respectively for the fermion, $f$, and, defining $\tau_{x}=\left(2 m_{x} / m_{h}\right)^{2}$, the loop functions are given by [26],

\footnotetext{
${ }^{2}$ The factor 5 in front of the scalar loop contribution $\mathcal{A}_{S}\left(\tau_{5}\right)$ arises from the sum of the singly and doubly charged scalar contributions from the custodial fiveplet.
}

$$
\begin{aligned}
\mathcal{A}_{W}\left(\tau_{W}\right) & =2+3 \tau_{W}+3 \tau_{W}\left(2-\tau_{W}\right) f\left(\tau_{W}\right), \\
\mathcal{A}_{f}\left(\tau_{f}\right) & =-2 \tau_{f}\left[1+\left(1-\tau_{f}\right) f\left(\tau_{f}\right)\right], \\
\mathcal{A}_{S}\left(\tau_{i}\right) & =-\tau_{i}\left[1-\tau_{i} f\left(\tau_{i}\right)\right] \quad(i=3,5) .
\end{aligned}
$$

In Eq. (26) the function $f(\tau)$ is defined as

$$
f(\tau)= \begin{cases}{\left[\sin ^{-1}\left(\sqrt{\frac{1}{\tau}}\right)\right]^{2},} & \text { for } \tau>1, \\ -\frac{1}{4}\left[\log \left[\frac{1+\sqrt{1-\tau}}{1-\sqrt{1-\tau}}\right]-i \pi\right]^{2}, & \text { for } \tau \leq 1 .\end{cases}
$$




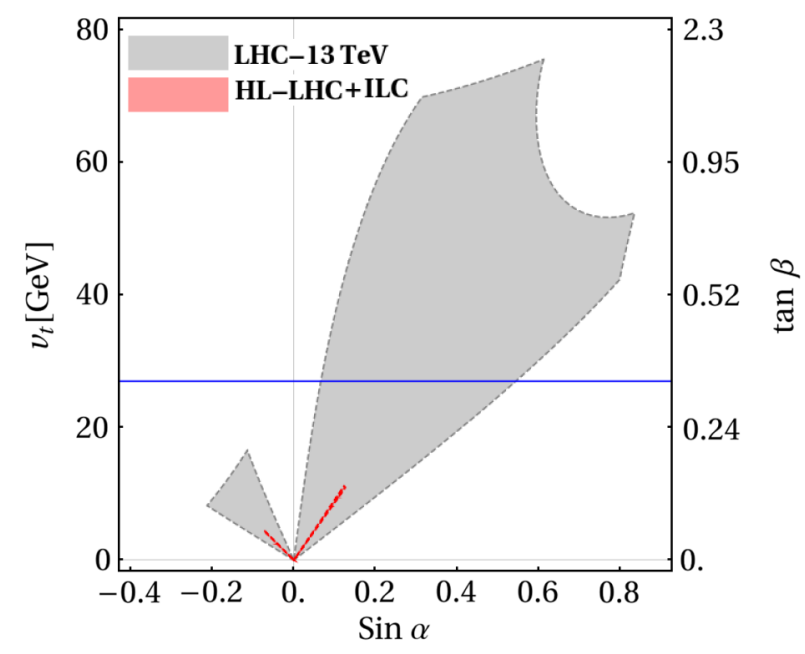

FIG. 3. Shrinking of the allowed region in Fig. 2 when combined with the constraints from $\kappa_{\gamma}$ in the limit $M_{1}$, $M_{2}=0$. The shaded regions in gray and red characterize the bounds arising from current [24] and future [25] measurements of $\kappa_{\gamma}$ respectively. In drawing this plot, a mass hierarchy $m_{3} \approx m_{5} \gg$ $m_{h}$ has been assumed. The horizontal line corresponds to the lower limit on $v_{t}$ arising from the theoretical constraints.

The expressions for $\kappa_{3}$ and $\kappa_{5}$ appearing in Eq. (25), which encapsulate the contributions from the charged scalars that are members of the custodial triplet and fiveplet respectively, are given $b^{3}$

$$
\begin{aligned}
& \kappa_{3}=\frac{M_{W}}{g m_{3}^{2}} g_{h H_{3}^{+} H_{3}^{-}}, \\
& \kappa_{5}=\frac{M_{W}}{g m_{5}^{2}} g_{h H_{5}^{+} H_{5}^{-}}=\frac{M_{W}}{g m_{5}^{2}} g_{h H_{5}^{++} H_{5}^{--}},
\end{aligned}
$$

where, $g$ is the usual $S U(2)_{L}$ gauge coupling and $g_{h H_{i}^{+(+)} H_{i}^{-()}}$denotes the trilinear coupling of $h$ with the $H_{i}^{+(+)} H_{i}^{-(-)}$pair. The loop functions in Eq. (26) saturate to finite nonzero values once the particles circulating in the loop become much heavier than $m_{h}$. Therefore, the (non) decoupling behaviors of the charged scalars will be captured by the quantities $\kappa_{3}$ and $\kappa_{5}$. In the limit $M_{1}, M_{2}=$ $0[4,5,27,28]$, we can calculate

$$
\begin{aligned}
\kappa_{3}= & \frac{-1}{2 m_{3}^{2}}\left[2 m_{3}^{2}\left(\cos \alpha \cos \beta+\frac{2 \sqrt{2}}{\sqrt{3}} \sin \alpha \sin \beta\right)\right. \\
& \left.+2 m_{h}^{2}\left(\frac{2 \sqrt{2}}{\sqrt{3}} \frac{\sin \alpha \cos ^{2} \beta}{\sin \beta}+\frac{\cos \alpha \sin ^{2} \beta}{\cos \beta}\right)\right],
\end{aligned}
$$

\footnotetext{
${ }^{3}$ Note that the equality $g_{h H_{5}^{+} H_{5}^{-}}=g_{h H_{5}^{++} H_{5}^{--}}$follows from the custodial symmetry.
}

$$
\begin{aligned}
\kappa_{5}= & \frac{-1}{2 m_{5}^{2}}\left[\frac{2 \sqrt{2}}{\sqrt{3}} \frac{\sin \alpha}{\sin \beta}\left(m_{h}^{2}+2 m_{5}^{2}\right)\right. \\
& \left.+m_{3}^{2}\left(6 \cos \alpha \cos \beta-4 \sqrt{6} \frac{\sin \alpha \cos ^{2} \beta}{\sin \beta}\right)\right] .
\end{aligned}
$$

Evidently, in the limit $m_{3} \approx m_{5} \gg m_{h}$, both $\kappa_{3}$ and $\kappa_{5}$ assume nonzero values, which implies that contrary to the common perception [12] the charged scalars do not decouple. Note that for $M_{1}, M_{2}=0$ but $v_{t} \neq 0$, the charged scalars receive their masses purely from the SSB sources and consequently, such nondecoupling behaviors are to be expected [29]. Because of this, the allowed region in Fig. 2 can be further constrained by superimposing the bounds from $\kappa_{\gamma}$, which has been shown in Fig. 3. From Fig. 3 we can easily see that, for $M_{1}, M_{2}=0$, using the projected combined accuracy for the HL-LHC and the ILC [25], we can restrict the upper limit on $v_{t}$ to be as low as $10 \mathrm{GeV}$ at 95\% C.L. From Fig. 3 it is also clear that the values of $\sin \alpha$ and $v_{t}$ can be strongly correlated if the measurement of $\kappa_{\gamma}$ agrees with the SM with the expected combined precision at the HL-LHC and the ILC. Consequently, the bound on $v_{t}$ will foster a strong upper limit on $\sin \alpha(|\sin \alpha|<0.1)$. Quite clearly, when combined with the lower limit on $v_{t}$ stemming from perturbative unitarity, we can potentially rule out the variants of GM models with $M_{1}, M_{2}=0$ using future Higgs precision data. Finally, in Fig. 4, we display the allowed parameter space at 95\% C.L. in the $m_{3}-m_{5}$ plane using the current as well as future precisions in $\kappa_{\gamma}$ for different sets of $\left\{\sin \alpha, v_{t}\right\}$ values. We find that as the accuracy in $\kappa_{\gamma}$ improves, the allowed region shrinks rapidly to the extent that with the future precision of about $1 \%$ at the HL-LHC and the ILC [25], the allowed area reduces to a thin sliver in the parameter space, thereby allowing us to strongly correlate $m_{3}$ and $m_{5}$ as well. In the same figure we also show how perturbative unitarity entails a different correlation in the $m_{3}-m_{5}$ plane (dark blue points) in the limit $M_{1}, M_{2}=0$. This implies that if the measurement of $h \rightarrow \gamma \gamma$ signal strength continues to agree with the corresponding SM expectation with higher precision, the GM models with $M_{1}, M_{2}=0$ will be increasingly disfavored. We have checked that the allowed region from $\kappa_{\gamma}$ in Fig. 4 does not crucially depend on the value of $v_{t}$ as long as it is nonzero.

To summarize, we have revisited the scalar sector of the GM model in the light of Higgs precision data. The GM model has long been preferred over minimal triplet model due to its attribution towards the preservation of custodial symmetry at the tree-level and thus lifting the stringent bound on the triplet scalar VEV. In this paper, we have demonstrated that, for certain variants of the GM models, the triplet VEV can still be tightly constrained using the Higgs precision data, especially the measurement of the $h \rightarrow \gamma \gamma$ signal strength. More specifically, we have shown that when the trilinear couplings in the scalar potential of 

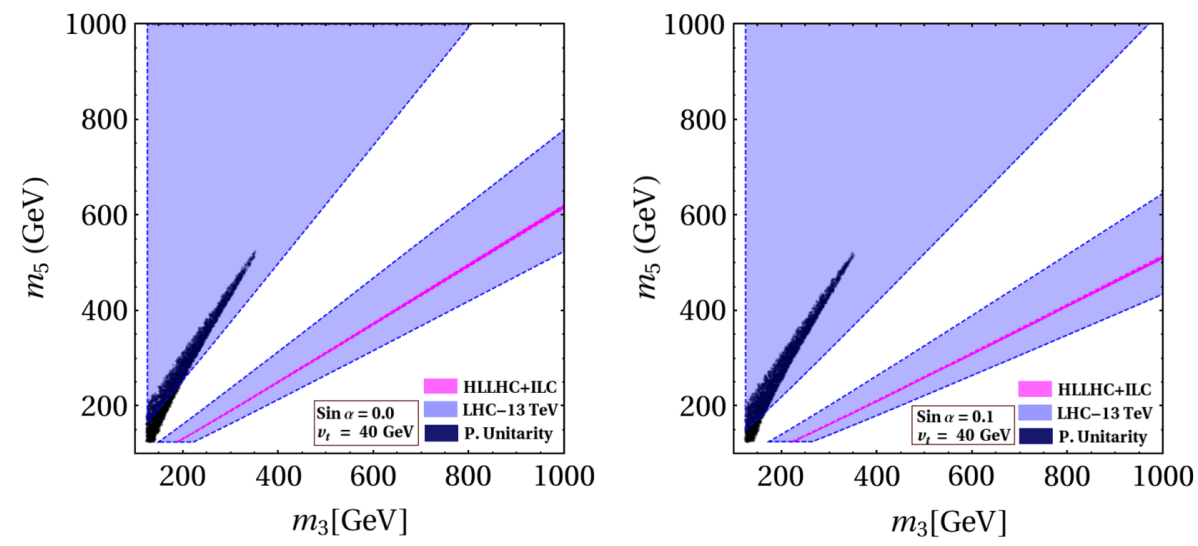

FIG. 4. Allowed regions at 95\% C.L. in the $m_{3}-m_{5}$ plane using the current (shaded in blue) [24] and projected (shaded in magenta) [25] accuracies in the measurement of $\kappa_{\gamma}$ for different sets of $\left\{\sin \alpha, v_{t}\right\}$ values. The dark blue points cover the region that passes the theoretical constraints of perturbative unitarity.

Eq. (3) vanish, the charged scalars do not decouple from the $h \rightarrow \gamma \gamma$ decay amplitude. Consequently, using the projected precision in the measurement of $\kappa_{\gamma}$, we have been able to set an upper limit on the triplet VEV, $v_{t}$, which can be as low as $10 \mathrm{GeV}$. This nondecoupling behavior of the charged scalars in the context of the GM model does not appear to be a widespread knowledge and therefore constitutes a new finding of our paper. The upshot of our analysis is that, unlike the bound on $v_{t}$ obtained using the flavor data $[7,8]$, our bounds derived from $\kappa_{\gamma}$ do not get washed away when the charged scalars are super heavy. We have also demonstrated how, using a precise measurement of $\kappa_{\gamma}$, the masses of the custodial triplet and fiveplet scalars can be correlated. Moreover, when these bounds are used in conjunction with the theoretical constraints from perturbative unitarity and stability, we have shown how the variants of GM models with $M_{1}, M_{2}=0$ can fall out of favor in the near future. Thus our current study highlights the importance of the measurement of Higgs to diphoton signal strength in the future experiments in the context of GM models.

\section{ACKNOWLEDGMENTS}

The work of DD has been supported by the Swedish Research Council, Contract No. 2016-05996.
[1] G. Aad et al. (ATLAS Collaboration), Observation of a new particle in the search for the standard model Higgs boson with the ATLAS detector at the LHC, Phys. Lett. B 716, 1 (2012).

[2] S. Chatrchyan et al. (CMS Collaboration), Observation of a new boson at a mass of $125 \mathrm{GeV}$ with the CMS experiment at the LHC, Phys. Lett. B 716, 30 (2012).

[3] H. Georgi and M. Machacek, Doubly charged Higgs bosons, Nucl. Phys. B262, 463 (1985).

[4] M. S. Chanowitz and M. Golden, Higgs boson triplets with $M(W)=M(Z) \cos \theta \omega$, Phys. Lett. 165B, 105 (1985).

[5] J. F. Gunion, R. Vega, and J. Wudka, Higgs triplets in the standard model, Phys. Rev. D 42, 1673 (1990).

[6] S. Kanemura and K. Yagyu, Radiative corrections to electroweak parameters in the Higgs triplet model and implication with the recent Higgs boson searches, Phys. Rev. D 85, 115009 (2012).

[7] K. Hartling, K. Kumar, and H. E. Logan, Indirect constraints on the Georgi-Machacek model and implications for Higgs boson couplings, Phys. Rev. D 91, 015013 (2015).
[8] A. Biswas, D. K. Ghosh, A. Shaw, and S. K. Patra, $b \rightarrow c \ell \nu$ anomalies in light of extended scalar sectors, arXiv: 1801.03375.

[9] H. E. Logan and M.-A. Roy, Higgs couplings in a model with triplets, Phys. Rev. D 82, 115011 (2010).

[10] S. Kanemura, M. Kikuchi, and K. Yagyu, Probing exotic Higgs sectors from the precise measurement of Higgs boson couplings, Phys. Rev. D 88, 015020 (2013).

[11] G. Belanger, B. Dumont, U. Ellwanger, J. F. Gunion, and S. Kraml, Global fit to Higgs signal strengths and couplings and implications for extended Higgs sectors, Phys. Rev. D 88, 075008 (2013).

[12] K. Hartling, K. Kumar, and H. E. Logan, The decoupling limit in the Georgi-Machacek model, Phys. Rev. D 90, 015007 (2014).

[13] C.-W. Chiang, S. Kanemura, and K. Yagyu, Novel constraint on the parameter space of the Georgi-Machacek model with current LHC data, Phys. Rev. D 90, 115025 (2014). 
[14] S. Kanemura, K. Tsumura, K. Yagyu, and H. Yokoya, Fingerprinting nonminimal Higgs sectors, Phys. Rev. D 90, 075001 (2014).

[15] C.-W. Chiang and K. Tsumura, Properties and searches of the exotic neutral Higgs bosons in the Georgi-Machacek model, J. High Energy Phys. 04 (2015) 113.

[16] H. E. Logan and V. Rentala, All the generalized GeorgiMachacek models, Phys. Rev. D 92, 075011 (2015).

[17] C.-W. Chiang, S. Kanemura, and K. Yagyu, Phenomenology of the Georgi-Machacek model at future electronpositron colliders, Phys. Rev. D 93, 055002 (2016).

[18] C.-W. Chiang, A.-L. Kuo, and T. Yamada, Searches of exotic Higgs bosons in general mass spectra of the Georgi-Machacek model at the LHC, J. High Energy Phys. 01 (2016) 120.

[19] J. Chang, C.-R. Chen, and C.-W. Chiang, Higgs boson pair productions in the Georgi-Machacek model at the LHC, J. High Energy Phys. 03 (2017) 137.

[20] B. Li, Z.-L. Han, and Y. Liao, Higgs production at future $e^{+} e$ colliders in the Georgi-Machacek model, J. High Energy Phys. 02 (2018) 007.

[21] M. Aoki and S. Kanemura, Unitarity bounds in the Higgs model including triplet fields with custodial symmetry, Phys. Rev. D 77, 095009 (2008).
[22] C.-W. Chiang and K. Yagyu, Testing the custodial symmetry in the Higgs sector of the Georgi-Machacek model, J. High Energy Phys. 01 (2013) 026.

[23] C. Degrande, K. Hartling, and H. E. Logan, Scalar decays to $\gamma \gamma, Z \gamma$, and $W \gamma$ in the Georgi-Machacek model, Phys. Rev. D 96, 075013 (2017).

[24] CMS Collaboration, Combined measurements of the Higgs boson's couplings at $\sqrt{s}=13 \mathrm{TeV}$, Tech. Rep. CMS-PASHIG-17-031, CMS, 2018.

[25] K. Fujii et al., Physics case for the international linear collider, arXiv:1506.05992.

[26] J. F. Gunion, H. E. Haber, G. L. Kane, and S. Dawson, The Higgs hunter's guide, Front. Phys. 80, 1 (2000).

[27] C. Englert, E. Re, and M. Spannowsky, Triplet Higgs boson collider phenomenology after the LHC, Phys. Rev. D 87, 095014 (2013).

[28] C. Englert, E. Re, and M. Spannowsky, Pinning down Higgs triplets at the LHC, Phys. Rev. D 88, 035024 (2013).

[29] G. Bhattacharyya and D. Das, Nondecoupling of charged scalars in Higgs decay to two photons and symmetries of the scalar potential, Phys. Rev. D 91, 015005 (2015). 